\title{
Use of Crumb Rubber Modified Binders and Asphalt Mixtures in Public Works Authority Road Projects - State of Qatar
}

\author{
Ezio Santagata \\ ezio.santagata@polito.it \\ Department of Environment, Land and Infrastructure Engineering, Politecnico di Torino, Turin, Italy \\ Haissam Sebaaly \\ h.sebaaly@anas.com.qa \\ ANAS S.p.A., Qatar Branch, Doha, Qatar \\ Osman Elhusain Mohamed Idris \\ osmane@ashghal.gov.qa \\ Quality and Safety Department, Public Works Authority (Ashghal), Doha, Qatar \\ Moaaz Hashim M. M. Ali \\ moaaz@ashghal.gov.qa \\ Quality and Safety Department, Public Works Authority (Ashghal), Doha, Qatar \\ Khalid Mohd I Al-Emadi \\ kemadi@ashghal.gov.qa \\ Quality and Safety Department, Public Works Authority (Ashghal), Doha, Qatar
}

\begin{abstract}
This paper illustrates the approach adopted by the Public Works Authority (Ashghal) of the State of Qatar for the widespread implementation in road projects of paving technologies related to the use of crumb rubber modified binders (CRMBs). Such an approach has entailed the monitoring of a full-scale preliminary trial, the definition of a prequalification system for crumb rubber and CRMB producers, and the development of mix design and quality control guidelines applicable to CRMB asphalt mixtures. Experimental results obtained in the preparatory phases of work and during the approval process of materials and mixtures are critically presented.
\end{abstract}

Keywords: Road pavements; Recycling; Crumb rubber; Asphalt; Mix design; Quality control

\section{INTRODUCTION}

The use of crumb rubber (CR) derived from end-of-life tires (ELTs) as a bitumen modifier has proven to be effective in enhancing the performance characteristics of asphalt mixtures worldwide (Way et. al., 2012). Currently available technologies fall within the category of the "wet processes" by means of which CR is directly incorporated in hot bitumen prior to mixing with aggregates, thereby leading to the formation of a binder characterized by enhanced elasticity and ductility (Bahia \& Davis, 1994). Depending upon the quantity of added CR and on the adopted mixing technique, binders may either be of the "asphalt rubber" type (with CR dosages higher than $15 \%$ by weight of total binder) or of the "terminal blend" type (with CR dosages of 8-12\%) (Bressi et al., 2019).

In the case of "asphalt rubber" binders, the CR-bitumen blend is cured in controlled conditions until the creation on the surface of CR particles of gel-like shells that are 
responsible for mutual interactions (Artamendi \& Khalid, 2006). The resulting binder is characterized by the presence of clearly visible CR particles and by a very high viscosity, thus leading to the need of either employing the binder immediately after production or keeping it in continuous agitation in properly equipped storage tanks. A further consequence of the peculiar structure of these binders is that their use is generally recommended in gap-graded or open-graded mixtures, which are characterized by the presence of voids in the mineral aggregates sufficient to include high binder volumes.

In case of "terminal blend" binders, a greater degree of digestion of the CR particles takes place in the bituminous medium, usually as a result of a finer grading (Way et al., 2012). The resulting binder is more homogeneous and, provided that its storage stability is initially evaluated, agitation prior to use may not be mandatory. Applications of these binders are similar to those of polymer-modified binders (PMBs), with the possibility of easily including them in dense-graded mixtures. It should be mentioned that the term "terminal blend" (which underlines the location of production) is frequently substituted by the more generic term, also adopted in this paper, "crumb rubber modified binder" (CRMB).

In the specific case of the State of Qatar, in the past there have been several full-scale applications involving the use of CRMBs. However, since these have not been organized as part of a general development program, their outcomes, which have been considered overall positive, cannot be analyzed on a quantitative basis. Furthermore, during the development of the road network, for infrastructures of strategic importance preference has been given to PMBs.

A renewed interest for the widespread implementation of CR technologies in Qatar has recently been stimulated by a scheme called "Ashghal Recycling Initiative", which has been launched by the Public Works Authority (Ashghal) in order to reduce construction costs, increase national self-sufficiency, limit environmental impacts, and enhance overall sustainability (Ashghal, 2018). Such an initiative has ultimately led to the enforcement of the use of CRMBs in lieu of PMBs for all projects overseen by the Road Projects Department (RPD) of Ashghal (RPD, 2018).

Implementation of CRMB technologies in Qatar has been supported by several activities carried out by the Quality \& Safety Department (QSD) of Ashghal and by ANAS S.p.A. Qatar Branch as part of the ongoing QA/QC Pavement Consultancy Services contract. In particular, these activities included the preliminary assessment of CR availability, the monitoring of a full-scale trial, and the preparation of two QSD Memoranda, respectively containing specific guidelines applicable to the production of CR and CRMBs (QSD, 2018a) and acceptance criteria to be adopted in the mix design and quality control of CRMB mixtures (QSD, 2018b).

This paper briefly summarizes the abovementioned activities, with the purpose of sharing obtained results and of illustrating the rationale underlying the official documents issued by Ashghal.

\section{AVAILABILITY OF CRUMB RUBBER}

According to available data, in the State of Qatar there is an estimated annual generation of approximately 1.5 million ELTs (QDB, 2017). These have been for a long time discarded as waste but have then become of major interest for local companies 
involved in their processing to produce $\mathrm{CR}$ which may be suitable for recycling.

As part of the preliminary activities of the QSD-ANAS team, the facilities of three different local CR producers were inspected, with the relevant assessment of employed technologies and potential productivity. It was found that the various plants combine sizereduction operations with other treatments (e.g. shredding, iron separation, granulation and sieving) in various configurations. However, all plants operate at "ambient" conditions, with no use of alternative techniques such as cryogenic milling or water jet-blasting. It is thus expected that resulting CR particles are irregular in shape and characterized by a significant surface roughness, which may lead to a high surface area available for interacting with bitumen in the production of CRMBs (Thodesen et al., 2009). All plants are capable of processing both truck tires, which are characterized by a higher content of natural rubber, and car tires.

Overall potential productivity of the plants was considered to be adequate for the local paving market, although it is envisioned that further improvements may be needed in the future in order to fully sustain the construction and maintenance of the road network.

\section{FEASIBILITY FULL-SCALE TRIAL}

In order to directly assess the local feasibility of CR recycling, a full-scale field trial was carried out on a local road, with the production and laying of a wearing course (WC) asphalt mix containing CRMB (50 mm thickness). For comparative purposes, the trial also included a WC asphalt mix with identical composition but containing PMB.

The RPD of Ashghal selected the trial location and organized construction operations. Given the preliminary nature of the activity, the involved Contractor was not required to perform a rigorous mix design of the CRMB mixture. Rather, reference was made to the Conformity Certificate of the mix containing gabbro aggregates and PMB (indicated as a PG76E-10). Target binder content for both mixtures was $3.9 \%$ by weight of total mix, while the target particle size distribution curve was of the continuous type, with $19.0 \mathrm{~mm}$ nominal maximum aggregate size.

The employed CRMB was produced by referring to the draft version of (QCS, 2018) (Section 6, Part 9), which was made available at the time of the trial. According to the manufacturer, the CRMB contained $11 \% \mathrm{CR}$ by weight of total binder and was graded as a PG76V-10. The CR used for modification, derived from car and truck tires, was of the -30 mesh type $(90-100 \%$ passing the 30 -mesh sieve, with a $600 \mu \mathrm{m}$ opening, and $100 \%$ passing the 20 -mesh sieve, with a $850 \mu \mathrm{m}$ opening).

Activities carried out by the QSD-ANAS team included critical observation of production and laying operations, and assessment of the most relevant characteristics of the produced mixtures. Experimental analyses were performed in the Ashghal Center for Research \& Development (ACRD).

\subsection{Composition and quality control testing}

Samples of loose mixtures taken on site were subjected to tests for the determination of their composition as per ASTM D2172-17 and ASTM D5444-15. Binder content was found to be very close to target for both produced mixtures. Results of grading analyses were in most part contained within tolerance ranges, with the only exception of filler content, which was slightly lower than expected and reflected into low values of 
the filler/bitumen ratio. Such a violation led to high values of the voids content, of the order of $9.0 \%$, both in Marshall specimens and in the field. Nevertheless, the study was continued since it was envisioned that a meaningful comparison between the mixtures could be obtained from performance-based testing (see section 3.2).

Encouraging results were obtained for the CRMB mixture with respect to its potential durability, captured by means of the tensile strength ratio (TSR) evaluated as per ASTM D4867-14. In fact, although the average TSR value was relatively low (equal to 70\%) as a result of the high voids content, it was identical to that which was found for the reference PMB mixture.

\subsection{Performance-based testing}

Performance-based testing of the two mixtures focused on the assessment of stiffness and resistance to permanent deformation. Specimens were compacted in the laboratory by means of different techniques (gyratory compaction as per AASHTO T312-15 and press box compaction as per AG:PT/T220) in order to reach a void content of $7.0 \pm 0.5 \%$.

Flexural stiffness tests were performed in the four-point bending configuration at $10{ }^{\circ} \mathrm{C}$ and $20^{\circ} \mathrm{C}$ by imposing a bending strain equal to 50 microstrain (as per AG:PT/ T274). Dynamic modulus tests were carried out in accordance with AASHTO T378-17 at three different temperatures $\left(4^{\circ} \mathrm{C}, 20^{\circ} \mathrm{C}\right.$ and $\left.45^{\circ} \mathrm{C}\right)$ and by considering three different stress states (corresponding to unconfined conditions and to confining pressures equal to $30 \mathrm{kPa}$ and $60 \mathrm{kPa}$ ). Given that test results were available at temperatures covering a sufficiently wide range, dynamic modulus data were modelled by means of the approach indicated in AASHTO R84-17. Resistance to permanent deformation was assessed by means of flow number tests (as per AASHTO T378-17) carried out at $45^{\circ} \mathrm{C}$.

Experimental results obtained from stiffness tests clearly indicated that the two mixtures exhibited a similar response under loading. In the case of flexural stiffness (Figure 1), such a similarity was quite remarkable: the maximum recorded percentage difference for all frequencies and temperatures was as low as 3.3\%, while the corresponding phase angle values were also very close to each other, with percentage differences never higher than $7.9 \%$. When considering the results of dynamic modulus tests, greater differences were observed between the two mixtures. In particular, the CRMB mixture exhibited higher stiffness and lower phase angle values at all frequencies and temperatures. The percentage difference increased by considering progressively lower frequencies, whereas it was quite variable as a function of temperature. Plots of the dynamic modulus and phase angle, shifted as per master curve construction, are shown in Figure 2, which also displays dynamic modulus master curves. Hence, it was confirmed that in the uniaxial loading mode the CRMB mixture exhibited a stiffer and more elastic response with respect to the PMB mixture, especially at lower frequencies and lower confining pressures. It should be underlined that the dynamic modulus requirement set by (QCS, 2014) for SUPERPAVE mixtures (minimum value of $1,920 \mathrm{MPa}$ at $45^{\circ} \mathrm{C}$ and $10 \mathrm{~Hz}$, no confining pressure) was satisfied by both mixtures, with values equal to 3,661 MPa and 2,810 MPa for the CRMB and PMB one, respectively. 

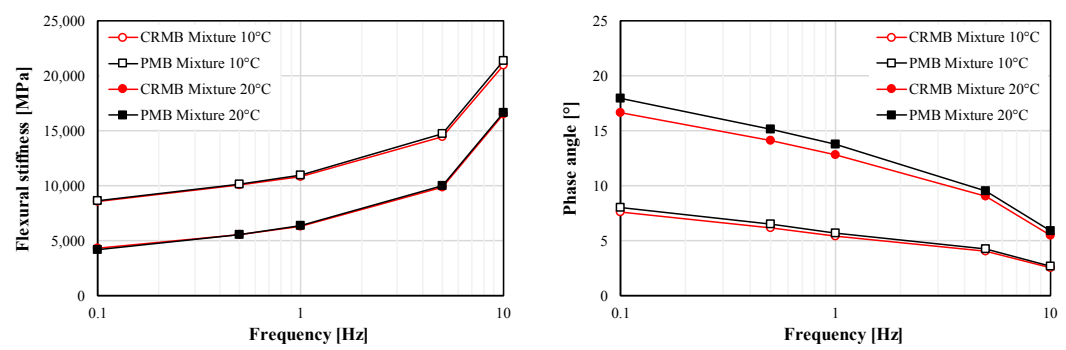

Figure 1: Results of flexural stiffness tests
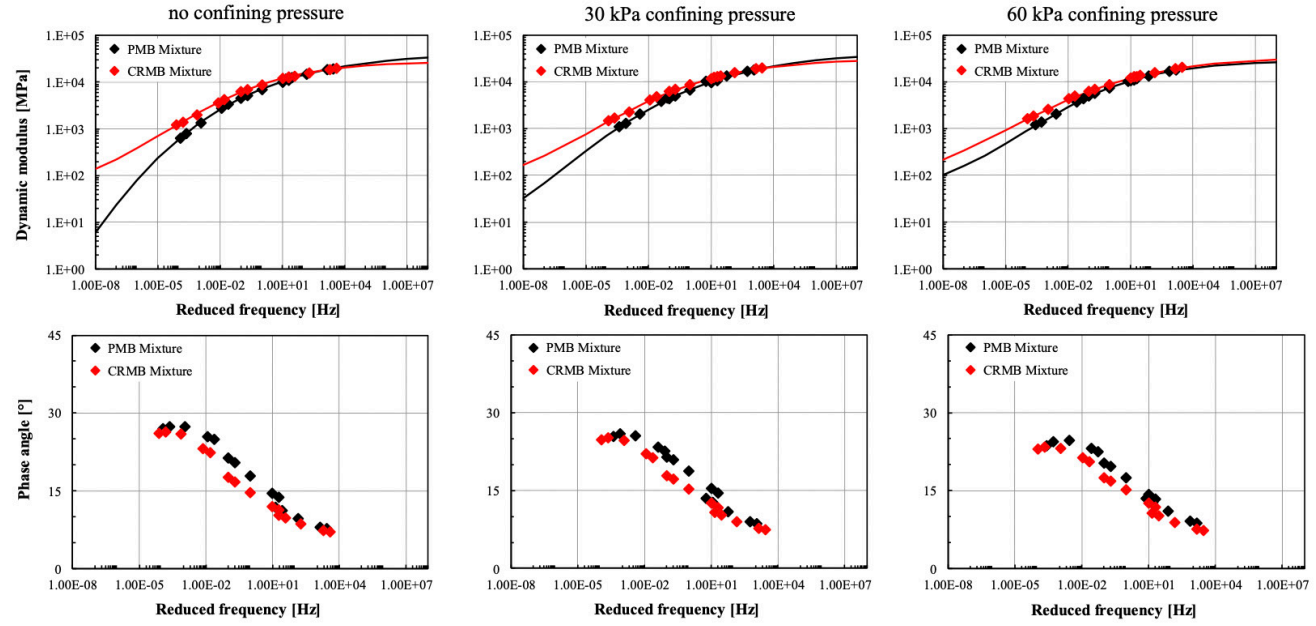

Figure 2: Dynamic modulus and phase angle master curves

Results obtained from permanent deformation tests are synthesized in Table 1, which contains average flow number values and corresponding void contents of specimens subjected to testing. Variation ranges of the measured parameters are also provided. Listed results clearly show that the two mixtures exhibited an equivalent resistance to permanent deformation. In particular, the difference between the average flow number values was equal to $3.4 \%$ and the corresponding variation ranges were found to overlap significantly. Finally, it should be emphasized that all flow number values well above the fixed acceptance threshold defined in (QCS, 2014) for the so-called SUPERPAVE mixes, equal to 740 at $45^{\circ} \mathrm{C}$.

Table 1: Results of flow number tests

\begin{tabular}{|c|c|c|c|c|}
\hline \multirow{2}{*}{} & \multicolumn{2}{|c|}{ CRMB Mixture } & \multicolumn{2}{c|}{ PMB Mixture } \\
\cline { 2 - 5 } & Average & Range & Average & Range \\
\hline Flow number (-) & 8,415 & $8,134-8,695$ & 8,140 & $7,014-9,386$ \\
\hline Voids content (\%) & 7.0 & $6.9-7.1$ & 6.8 & $6.7-6.9$ \\
\hline
\end{tabular}

Based on the results discussed above, it was concluded that CRMB mixtures, when compacted to target volumetrics, have the potential of being equivalent, and perhaps even superior, to PMB mixtures in terms of their load spreading capacity and resistance to rutting. However, in this stage it was clearly emphasized that further improvements were necessary in order to properly optimize the composition of CRMB mixtures. Thus, 
further work was initiated in the ACRD, and is still in progress, for the evaluation of the volumetric and mechanical characteristics of trial CRMB mixtures.

\section{QUALITY SYSTEM FOR CRMB MIXTURES}

As a result of the preliminary activities described in sections 2 and 3, and by considering the outcomes of ongoing testing in the ACRD, the QSD-ANAS team worked on the finetuning of the quality system to be locally adopted for CRMB mixtures. In particular, the existing Ashghal QA/QC system was supplemented with the two previously mentioned QSD Memoranda (QSD, 2018a-b). The contents of these documents, prepared in line with (QCS, 2014) and with the draft of the upcoming (QCS, 2018), are synthesized in the following, with a discussion of key issues which should be addressed by all involved Parties (producers, consultants, contractors, third party laboratories).

\subsection{Prequalification of CR producers}

The purpose of the prequalification requirements made mandatory in the QSD Memorandum (QSD, 2018a) is to ensure the consistent production of high-quality CR, capable of acting as an efficient bitumen modifier. In such a context, constraints are imposed to CR particle size distribution and to its cleanliness, with a minimum quality control testing frequency of one determination (of each relevant parameter or quantity) for every 15 tons of production. The corresponding sampling methodology has been drawn from the experience of the California State Transportation Agency.

With respect to CR grading, only fine products of the -30 mesh type are allowed for use since they can be dispersed in bitumen more homogeneously, thereby reducing segregation potential. Furthermore, fine CR is more suitable for mixtures as those adopted in Qatar, characterized by a dense aggregate packing and by a rather low binder content.

Although the QSD Memorandum does not introduce any gradation acceptance band, $\mathrm{CR}$ producers are requested to report the full particle size distribution by referring to a subset of the ASTM D5644-18 series (30-mesh, 40-mesh, 80-mesh, 200-mesh and 400-mesh). This is due to the fact that surface area is highly affected by finer particles, and it is envisioned that in the future specific measurements of such a parameter may be introduced in the prequalification stage for a better control of CR quality (Santagata et. al., 2012).

With respect to CR cleanliness, various parameters need to be monitored throughout production (by following prescribed procedures) and continuously compared to allowable maximum thresholds or acceptance ranges. Relevant parameters include specific gravity and content of moisture, non-ferrous and ferrous metal particles, fibers, and foreign contaminant materials. It should be noted that most of the requirements have been drawn from those contained in ASTM D6114-19, which have been extensively validated for the assessment of CR products employed for asphalt rubber production.

It should be underlined that there are no restrictions on CR origin, since the QSD Memorandum allows the use of products derived from car and/or truck tires. However, producers need to clearly declare the origin of processed ELTs.

When considering the applications received by QSD since the issue of the Memorandum, it was observed that all local CR producers were able to meet the requirements synthesized above. 


\subsection{Prequalification of CRMB producers}

In line with the high standards required for the prequalification of all manufacturers involved in the pavement construction supply chain, the QSD Memorandum (QSD, 2018a) requires CRMB producers to give full evidence of their quality management system and provide relevant information on their production plant and staff. As in the case of $\mathrm{CR}$, prequalification requirements have the function of ensuring the consistent production of uncontaminated high-quality CRMBs, which have to possess rheological properties that match the prevalent loading and environmental conditions occurring in road pavements in the State of Qatar. Required quality control testing entails the verification of set requirements on each batch, defined as the quantity of binder produced and stored in one tank once the production run into that tank has been completed.

CRMB producers are required to clearly state the source of employed CR, providing all the necessary supporting documents, and to declare the adopted modification dosage, which should be comprised between $10 \%$ and $25 \%$. While the lower limit is consistent with the usual formulation of terminal blend binders, the upper one, more typical of asphalt rubber products, allows producers to develop more advanced formulations, thus including the potential introduction of improved technologies which may lead to a reduction of bitumen consumption and to an increase of the cost-effectiveness of production. As a result of the performance-based nature of acceptance requirements, no tests are necessary for the verification of CR dosage. Nevertheless, this type of analysis may be taken into consideration in the future for a critical evaluation of construction costs and in the context of Green Public Procurement policies and of Life Cycle Analyses (Farina et al., 2017). This will require specific research work in the ACRD, which will be carried out by considering relevant investigations documented in literature (Zanetti et al., 2018).

Although purity requirements are already introduced in the $\mathrm{CR}$ prequalification process, additional verifications, to be carried out directly on the CRMB, were deemed necessary. These were expressed by referring to the outcomes of a two-step laboratory procedure which combines binder solubility (as per ASTM D5546-09 or AASHTO T4414) and burning with an ignition oven at $400{ }^{\circ} \mathrm{C}$ for 1 hour. The maximum allowable weight of the resulting residue, equal to $1 \%$ of the original sample, ensures the absence of contaminants and is consistent with CR requirements and typical CRMB formulations. Furthermore, assessment of the residue after the solubility test may provide information on the degree of digestion of the CR particles during production, thereby allowing a critical assessment of the adopted production technology.

With respect to rheological properties and to the corresponding SUPERPAVE grading, it is well known that the climate encountered in Qatar calls for the use of binders belonging to performance grade PG76-10 (Al-Abdul Wahhab et al., 1997). QCS (2014) contains such a requirement for PMBs, by referring to the original SUPERPAVE scheme implemented in AASHTO M 320-17 and by considering the application of the more recent scheme of AASHTO M 332-14 as an option.

The QSD Memorandum refers to AASHTO M 332-14 for the assessment and grading of CRMBs. However, it removes the requirements therein contained for solubility, nonrecoverable creep compliance difference ( $\mathrm{J}_{\text {nrdiff }}$, which needs only to be reported), and viscosity (once again only to be reported). These modifications to the specification 
scheme are respectively due to the specific nature of CRMBs (characterized by the presence of insoluble, not completely digested rubber residues), to the specific non-linear response under oscillatory loading (which makes the current $\mathrm{J}_{\text {nrdiff }}$ limits not applicable) and to the use of efficient pumping systems in asphalt mix production (which can handle high viscosity binders). Furthermore, in order to account for the presence of undigested rubber particles in the binder, rheological tests for binder grading carried out in the oscillatory mode (as per AASHTO T315-10 and AASHTO T350-19) are required to be conducted with an increased measurement gap of $2 \mathrm{~mm}$. Such a prescription is consistent with current research and has been proven to be effective for the reliable assessment of the rheology of CRMBs.

It should be underlined that the QSD Memorandum explicitly requires CRMBs to be stored in continuously agitated tanks, both at the modification terminal and the asphalt plant. Such a constraint was deemed necessary regardless of CR dosage in order to avoid any risk of segregation, which may jeopardize the successful implementation of CRMB technologies.

Results documented in the applications submitted to QSD for approval indicate that the CRMBs produced by two different manufacturers (containing CR only from truck tires) easily met all the requirements synthesized above. In particular, they were all reported to be PG76E-10 and exhibited viscosity values at $135{ }^{\circ} \mathrm{C}$ which satisfied the waived requirement of AASHTO M 332-14.

\subsection{Mix design and quality control of CRMB mixtures}

The QSD memorandum which focuses on the mix design and quality control of CRMB asphalt mixes (QSD, 2018b) is consistent with QCS 2014. However, additional requirements have been introduced in order to account for the particular nature and behavior of CRMBs.

With respect to mix design, it is left to the Contractor to decide whether to adopt the classical Marshall method, or to adopt the more advanced SUPERPAVE approach. In both cases, as for all other mixtures certified for use in Qatar, approval of the mix design requires a preliminary laboratory study followed by trial batching and trial laying. Quality control is carried out by referring to the sampling and testing frequencies listed in (QCS, 2014), supplemented by further indications which apply to the newly introduced tests described in the following.

Requirements referred to in both the mix design and quality control process are taken from (QCS, 2014), considering those defined for PMB mixtures as prevalent (if present). Additional acceptance limits are introduced for Marshall-designed mixtures with respect to the results of Indirect Tensile Strength (ITS) tests carried out at $25^{\circ} \mathrm{C}$, before and after static water immersion (as per ASTM D4867-09). Furthermore, in the case of all mixtures (Marshall- and SUPERPAVE-designed), reference is made to the results of Hamburg wheel-track (HWT) tests carried out on water-submerged specimens (as per AASHTO T324-19). It should be clarified that rather than for the assessment of moisture sensitivity, these procedures were selected for the evaluation of strength and resistance to permanent deformation in extreme conditions, thereby ensuring the identification of durable mixes regardless of the environment in which they are placed. Thus, a minimum TSR value of $75 \%$, slightly lower than those adopted in other specifications, was considered adequate 
for such a purpose in the case of Marshall mixtures. The standard limiting maximum rut depth of $12.5 \mathrm{~mm}$ was considered for HWT tests: these were preferred to flow number tests in accordance with current international trends.

Results obtained in the prequalification of four wearing course CRMB asphalt mixtures approved by QSD are synthesized in Table 2, where they compared to the acceptance limits. All Contractors chose the Marshall design option. In the mix design phase tests were carried out only by third party laboratories, whereas in the plant verification phase tests were also performed by the ACRD. All mixtures contained gabbro aggregates with a similar continuous total grading (not displayed in this paper).

Table 2: Results of mix design tests (Marshall-designed wearing course asphalt mixtures)

\begin{tabular}{|c|c|c|c|c|c|c|c|c|c|}
\hline & & Mix & re\#1 & Mixt & re\#2 & Mix & e \#3 & Mix & e\#4 \\
\hline & $\begin{array}{l}\text { Acceptance } \\
\text { limits }\end{array}$ & $\begin{array}{c}\text { Mix } \\
\text { design }\end{array}$ & $\begin{array}{c}\text { Plant } \\
\text { trial }\end{array}$ & $\begin{array}{c}\text { Mix } \\
\text { design }\end{array}$ & $\begin{array}{c}\text { Plant } \\
\text { trial }\end{array}$ & $\begin{array}{c}\text { Mix } \\
\text { design }\end{array}$ & $\begin{array}{c}\text { Plant } \\
\text { trial }\end{array}$ & $\begin{array}{c}\text { Mix } \\
\text { design }\end{array}$ & $\begin{array}{c}\text { Plant } \\
\text { trial }\end{array}$ \\
\hline Optimum binder content (OBC), \% & $3.4-4.4$ & 4.1 & 4.1 & 4.2 & 4.1 & 4.0 & 4.0 & 4.0 & 4.2 \\
\hline Air voids $(\mathrm{Va}), \%$ & $5.0-8.0$ & 6.2 & 7.2 & 5.9 & 6.5 & 5.9 & 6.3 & 6.0 & 6.3 \\
\hline Voids in mineral aggregate (VMA), $\%$ & $\geq 14.0$ & 14.6 & 15.6 & 15.8 & 15.4 & 15.5 & 14.3 & 15.1 & 14.9 \\
\hline Voids filled with asphalt (VFA), $\%$ & $50-75$ & 58 & 54 & 62 & 58 & 59 & 56 & 60 & 58 \\
\hline Marshall stability (Sta), $\mathrm{kN}$ & $\geq 13.0$ & 16.8 & 13.5 & 15.7 & 18.5 & 19.5 & 16.9 & 17.3 & 15.3 \\
\hline Marshall flow $(\mathrm{F}), \mathrm{mm}$ & $2.0-4.0$ & 2.9 & 2.5 & 2.7 & 2.5 & 2.6 & 2.64 & 2.3 & 2.8 \\
\hline Marshall quotient (Sta/F), $\mathrm{kN} / \mathrm{mm}$ & $\geq 5.25$ & 5.69 & 5.40 & 5.86 & 7.40 & 7.5 & 6.40 & 7.3 & 5.46 \\
\hline Air voids at 400 blows per face, $\%$ & $\geq 4.0$ & 4.2 & 4.4 & 4.4 & 4.5 & 4.4 & 4.1 & 4.5 & 4.9 \\
\hline Retained stability, \% & $\geq 75$ & 89 & 94 & 86.9 & 90 & 84 & 85 & 96 & 83 \\
\hline Tensile strength ratio (TSR), $\%$ & $\geq 75$ & 78 & 81 & 77 & 84 & 78 & 79 & 76 & 81 \\
\hline Filler to bitumen ratio ( $\mathrm{F} / \mathrm{OBC})$ & $0.75-1.35$ & 1.03 & 0.95 & 0.90 & 0.76 & 1.10 & 1.25 & 1.07 & 1.05 \\
\hline Rut depth from HWT test, mm & $\leq 12.5$ & 6.6 & 4.7 & 5.6 & 7.2 & 3.2 & 3.7 & 4.3 & 3.8 \\
\hline
\end{tabular}

From the analysis of the data listed in Table 2 it can be observed that the considered mixtures were characterized by similar optimum binder content values (comprised in the 4.0-4.2 range), close to those of typical PMB wearing course mixtures employed in Qatar. As a consequence of such a similarity (and of the previously mentioned similarity in aggregate grading), volumetric and mechanical characteristics of Marshall-compacted specimens were also contained in very narrow ranges. All mixtures were characterized by a dense aggregate packing (revealed by the contained values of the voids in the mineral aggregate, VMA) combined with a sufficiently limited volume of binder (indicated by the voids filled with asphalt, VFA), which led to satisfactory mechanical properties. It is interesting to underline that the newly introduced TSR and HWT requirements were met, with average values of corresponding parameters equal to $79.3 \%$ and $4.9 \mathrm{~mm}$. Only for one mix (\#4) use was made of an anti-stripping agent, employed with a dosage of $0.5 \%$ by weight of binder.

\section{CONCLUSION}

Based on the discussion of the activities presented in this paper, it can be concluded that implementation of CRMB technologies in Qatar is proceeding in accordance with the plans of the "Ashghal Recycling Initiative". Local CR and CRMB producers have shown to be capable of meeting the recently issued prequalification requirements and several contractors have already obtained official approval of their asphalt mix designs. Ashghal will seek further improvements in the future, by continuously supporting the application of proposed quality control measures and by sustaining research activities carried out in the ACRD. 


\section{REFERENCES}

Al-Abdul Wahhab, H. I., Asi, I. M., Al-Dubabe, I. A. \& Farhat Ali, M. (1997). Development of performance-based bitumen specifications for the Gulf countries. Construction and Building Materials, 11(1), 15-22.

Artamendi, I. \& Khalid, H. A. (2006). Diffusion kinetics of bitumen into waste tire rubber. Journal of the Association of Asphalt Paving Technologists, 75, 133-164.

Ashghal (2018). Roadmap for implementation of Ashghal Initiative of recycling in construction projects (Circular No (69)). Doha, Qatar, 09/05/2018.

Bahia, H. U. \& Davis, R. (1994). Effect of crumb rubber modifiers (CRM) on performance-related properties of asphalt binders. Journal of the Association of Asphalt Paving Technologists, 63, 414-449.

Bressi, S., Fiorentini, N., Huang, J. \& Losa, M. (2019). Crumb rubber modifier in road asphalt pavements: state of the art and statistics. Coatings, 9(6), Article number 384.

Farina, A., Zanetti, M. C., Santagata, E. \& Blengini, G. A. (2017). Life cycle assessment applied to bituminous mixtures containing recycled materials. Resources, Conservation \& Recycling, $117,204-212$.

Qatar Construction Specifications (QCS) (2014). Ministry of Municipality and Environment, Doha, Qatar.

Qatar Development Bank (QDB) (2017). Materials Recovery, Doha, Qatar.

Quality and Safety Department (QSD), Ashghal (2018a). Pre-qualification of Crumb Rubber and Crumb Rubber Modified Binder Producers, Doha, Qatar, 13/12/2018.

Quality and Safety Department (QSD), Ashghal (2018b). Guidelines for Mix Design and Quality Control of CRMB Asphalt Mixes, Doha, Qatar, 18/11/2018.

Road Projects Department (RPD), Ashghal (2018). Use of Crumb Rubber in RPD projects (Circular No. (26)). Doha, Qatar, 28/10/2018.

Santagata, E., Dalmazzo, D., Lanotte, M., Zanetti, M. C. \& Ruffino, B. (2012). Relationship between crumb rubber morphology and asphalt rubber viscosity. Proceedings, $5^{\text {th }}$ Asphalt Rubber Conference, Munich, Germany, 513-532.

Thodesen, C., Shatanawi, K. \& Amirkhanian, S. (2009). Effect of crumb rubber characteristics on crumb rubber modified (CRM) binder viscosity. Construction and Building Materials, 23, 295-303.

Way, G. B., Kaloush, K. E. \& Biligiri, K. P. (2012). Asphalt-rubber standard practice guide, Rubber Pavements Association, Tempe, Arizona, the USA.

Zanetti, M. C., Ruffino, B., Santagata, E., Dalmazzo, D. \& Lanotte, M. (2018). Determination of crumb rubber content of asphalt rubber binders. Journal of Materials in Civil Engineering, 30 (4), Article number 04018041, 1-6. 